\title{
Performance Evaluation of MIMO-OFDM Schemes in Presence of Carrier Frequency Offset (CFO)
}

\author{
Ankita K. Panchal ${ }^{1}$ \\ Assistant Professor, EC Department, Sigma Group of Institutes, Vadodara, India ${ }^{1}$
}

\begin{abstract}
Wireless designers constantly seek to improve the data rate, spectral efficiency, coverage of wireless networks and link reliability. Orthogonal Frequency Division Multiplexing (OFDM) technique has been widely accepted for the transmission of high rate data. Multiple Input Multiple Output (MIMO) techniques provide strong diversity gain and can increase the link throughput with the use of Adaptive Modulation and Coding (AMC). The idea behind AMC is to dynamically adapt the modulation and coding scheme to the channel conditions so as to achieve the highest spectral efficiency at all times. Further MIMO schemes can be combined with OFDM as STBC-OFDM or SFBC-OFDM. Combining the advantages of space-time coding and OFDM is attractive in wireless system designs. However, one of the main drawbacks is its high sensitivity to frequency offsets since the orthogonality between sub carriers is destroyed thus leading to considerable system performance degradation. The Carrier Frequency Offset (CFO) arises mainly due to the Doppler shift and/or oscillator mismatch. Hence, the effect, estimation and correction of frequency offsets have been subject of intensive research in the decade. Performance of various MIMO techniques is evaluated with combination of OFDM. The objective is optimum combination of MIMO-OFDM in presence of CFO. This system is also implemented as per WiMAX standard. The performance is also observed with different modulation techniques and multiple antenna system for multi-users.
\end{abstract}

Keywords: Carrier frequency offset (CFO); Orthogonal Frequency Division Multiplexing OFDM; Multiple Input Multiple Output (MIMO); Space Time Block Code-OFDM (STBC-OFDM); Space Frequency Block Code-OFDM (SFBC-OFDM)

\section{INTRODUCTION}

Wireless technology has quickly become part of everyday Orthogonal Frequency DivisionMultiplexing (OFDM), life. Wireless LANs, cell phone networks, and personal combined with MIMO techniques performs significantly area networks are just a few examples of widely used in multipath fading environment. OFDM technique has wireless networks. However, wireless devices are limited been widely accepted for the transmission of high rate to range and data rate. During the last decade, tremendous data. OFDM has been adopted as the modulation method efforts are made to improve capacity of wireless networks of choice for practically all the new wireless technologies like wireless local area network and cellular network. In being used and developed today. But it is observed that particular, compared to only improvement in the data rates OFDM is highly affected by Carrier Frequency Offset made available by today's technology, the need for (CFO). Frequency offset, which could arrive due wireless internet access and multimedia applications tooscillator instabilities, or relative motion require an increase in information throughput with order betweentransmitter and receiver has the potential to of magnitude. The research community has spent a great destroy the orthogonality and hence the optimality of the deal of effort in finding ways to overcome these corresponding receiver. MIMO has been incorporated into limitations. One major technological breakthrough that many recently developed wireless systems like various will make this increase in data rate possible is the use of WLAN IEEE802.11n standard, WiMAX IEEE802.16e multiple antennas at the transmitters and receivers in the and 3GPP Long Term Evolution. WiMAX is one of the system. In the early 1990s, development of transmit diversity techniques has started.

Since then the interest in the topic has grown in a rapid fashion. In fact, we can expect Multiple-Input MultipleOutput (MIMO) technology to be a cornerstone of many wireless communication systems due to the potential increase in data rate and performance of wireless links offered by transmit diversity and MIMO technology. MIMO is the current theme for the international wirelessresearch. Transmit diversity consists of transmitting a single space-time coded stream through all antennas. most popular next generation technologies available at the time of writing, aiming to provide high-speed broadband wireless access. As a standardized technology, WiMAX ensures the interoperability of equipment certified by the WiMAX Forum, resulting in a significant cost reduction for service providers that would like to use products manufactured by diverse vendors. This distinct advantage has paved the way for global broadband wireless services. Another key benefit of WiMAX is that it has been optimized for offering excellent Non-Line-of-Sight (NLOS) coverage with the aid of advanced wireless transmission techniques, such as MIMO transmit/receive 
combined with OFDM. MIMO-OFDM is evaluated as per the WiMAX standard.

II. OVERVIEW OF OFDM, MIMO AND MIMOOFDM

The requirement for wide bandwidth and flexibility imposes the use of efficient transmission methods that would fit to the characteristics of wideband channels especially in wireless environment where the channel is very challenging. In wireless environment the signal is propagating from the transmitter to the receiver along number of different paths, collectively referred as multipath. The research community has spent a great deal of effort on finding ways to overcome these limitations. One method is to use Multiple-Input Multiple-Output (MIMO) links. The multiple antennas allow MIMO systems to perform diversity coding (space-time coding). MIMO is the use of multiple antennas at both the transmitter and receiver to improve communication performance. It is one of several forms of smart antenna technology.

\section{A. Orthogonal Frequency Division Multiplexing}

OFDM is a broadband multicarrier modulation method that offers superior performance and benefits over older, more traditional single-carrier modulation methods because it is a better fit with today's high-speed data requirements. OFDM has been adopted as the modulation method of choice for practically all the new wireless technologies being used and developed today. It is perhaps the most spectrally efficient method discovered so far, and it mitigates the severe problem of multipath propagation that causes massive data errors. Fig.2.1 shows the OFDM block diagram. Serial to parallel converter collects $\mathrm{K}$ serial data symbols X (m) into a data block or vector X (n). X (n) is modulated by an IDFT into OFDM symbol vector $\mathrm{X}$ (n). A length $G$ cyclic prefix is added to $X$ (n) and transmitted through a frequency-selective channel $h(n)$ of order L. The reasons to use a cyclic prefix for the guard interval are to maintain the receiver carrier synchronization. Some signals instead of a long silence must always be transmitted cyclic convolution can still be applied between the OFDM signal and the channel response to model the transmission system. The transmitted signal is given by: $x_{0}(n)=\frac{1}{\sqrt{N}} \sum_{k=0}^{N-1} X_{0}(k) e^{e j 2 \pi n k / N}$, here $\mathrm{n}$ ranges fromN $\mathrm{g}_{\mathrm{g}}$ to $\mathrm{N}-1$.

At the receiver, the cyclic prefix is removed from the received signal and the remaining signal is demodulated by a DFT into $\mathrm{Y}(\mathrm{n})$. Down converting RF introduces Carrier frequency offsets $\Delta \mathrm{f}$ into received signal. Besides the noise component, the demodulated symbol $\mathrm{Y}(\mathrm{n}, \mathrm{k})$ is just the product of the complex gain and the corresponding data symbol $X(n, k)$. OFDM with a cyclic prefix transforms a frequency-selective fading channel into $\mathrm{K}$ decoupled and perfectly at fading sub channels.

$$
\begin{aligned}
& \text { The received signal is given } \\
& y_{0}(n)=\frac{1}{\sqrt{N}} \sum_{k=0}^{N-1} X_{0}(k) e^{e j 2 \pi n(k+\varepsilon) / N}+z_{0}(n) .
\end{aligned}
$$

where $\varepsilon=\Delta \mathrm{f} \mathrm{T}$ is the normalized CFO of the system and $\mathrm{z}_{0}(\mathrm{n})$ denotes the AWGN samples.

where $\varepsilon=\Delta \mathrm{f} \mathrm{T}$ is the normalized CFO of the system and $\mathrm{z}_{0}(\mathrm{n})$ denotes the AWGN samples.

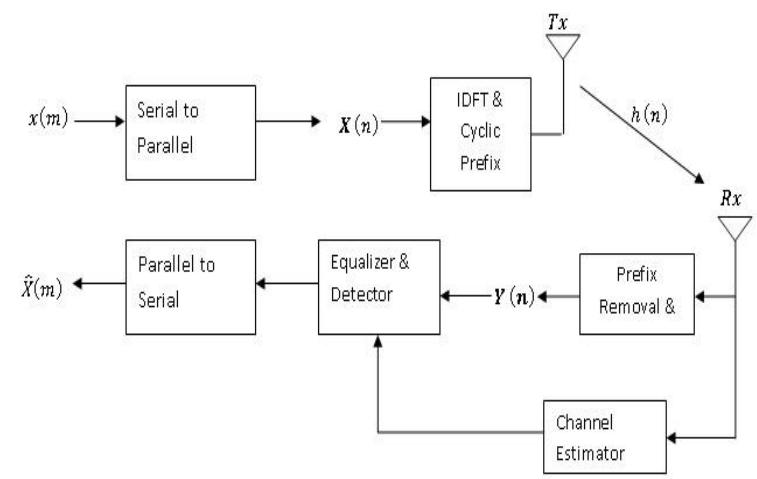

Fig2.1: OFDM Block Diagram

\section{B. MIMO-OFDM System}

MIMO wireless systems have captured the attention of international standard organizations. The use of MIMO has been proposed multiple times for use in the high-speed packet data mode of third-generation cellular systems as well as the fourth-generation cellular systems. Frequency selective fading is a dominant impairment in mobile communications. Fading reduces receive Signal-to-Noise Ratio (SNR) and degrades the Bit-Error-Rate (BER). Frequency selectivity of the channel, i.e. delay spread, induces carrier frequency offset. To combat frequencyselective fading, diversity techniques are used. Space-time block coding has emerged as an efficient means of achieving near optimal transmitter diversity gain. Orthogonal frequency division multiplexing (OFDM) with a sufficiently long cyclic prefix can convert frequencyselective fading channels into multiple fading subchannels. Combined space-time block code and OFDM can give better performance. There are two major coding approaches for MIMO-OFDM systems. One is the Space Frequency (SF) coding approach, where coding is applied within each OFDM block to exploit the Spatial and Frequency diversities. The other one is the Space Time Frequency (STF) coding approach, where the coding is applied across multiple OFDM blocks to exploit the spatial, temporal, and frequency diversities.

\section{B.1 Alamouti Space-Time Code-OFDM [1]}

Alamouti scheme is the first space-time block code scheme that provides full transmit diversity for systems with two transmit and one receive antennas. It is a unique scheme in which it is the only space-time block code with a $\mathrm{N} X \mathrm{~N}$ complex transmission matrix to achieve the full rate of one. Fig2.2.1 represents the Alamouti STBC block diagram. At the transmitter side, a block of two symbols are taken from the source data and sent to the modulator. After that, Alamouti space-time encoder takes the two modulated symbols, $s_{1}$ and $s_{2}$ at a time and creates $G_{2}$ encoding matrix $S$ where the symbols $s_{1}$ and $s_{2}$ are mapped to two transmit antennas in two transmit times as defined in the following: Space-time encoder takes two data vectors $X(n)$ and $X(n+1)$ and transmits 
Antenna 1 :

$X(n)$

Antenna 2 :
$X^{*}(N+1)$ $-X^{*}(N+1)$

where the symbol * represents the complex conjugate. Therefore, $X^{*}(n)$ is the complex conjugate of $X(n)$. The encoder outputs are transmitted in two consecutive transmission periods from the two transmit antennas. IDFT of symbols takes place and cyclic prefix is added to it. The symbols are transmitted through channel $h_{1}$ and $h_{2}$ as follows:

$$
\begin{aligned}
& h_{1}(t)=h_{1}(t+T)=h_{1}=\left|h_{1}\right| e^{j \theta_{1}} \\
& h_{2}(t)=h_{2}(t+T)=h_{2}=\left|h_{2}\right| e^{j \theta_{2}}
\end{aligned}
$$

where $\left|h_{1}\right|$ and $\theta_{i}, \mathrm{i}=1,2$ are the amplitude gain and phase shift for the path from transmit antenna I to the receive antenna and $\mathrm{T}$ is the symbol duration. At the receiver the reverse process is carried out. The symbols are detected through maximum likelihood detection and received at the receiver:

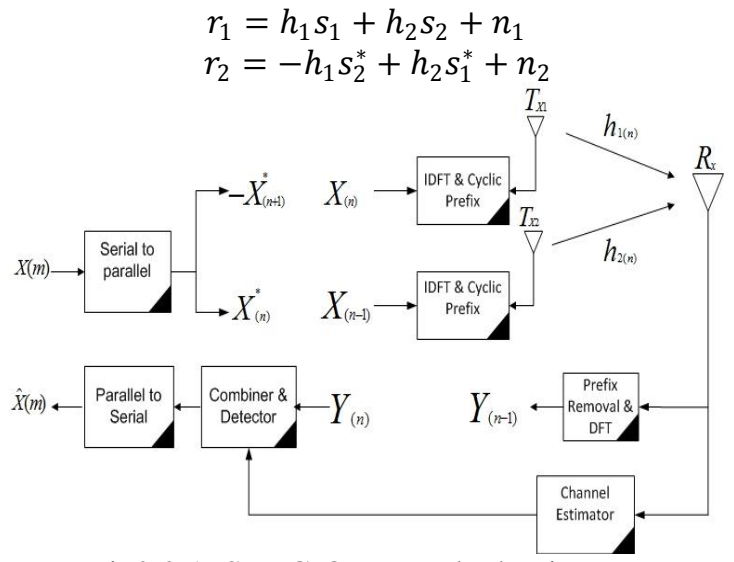

Fig2.2.1: STBC-OFDM Block Diagram

\section{B.2 Space Frequency Block Code-OFDM}

The block diagram of space frequency block coding is shown in Fig.2.2.2. Sequences of $\mathrm{N}_{c}$ time and frequency interleaved data symbols $X_{n}$ are transmitted in one OFDM symbol. The data symbols are interleaved by the block before space frequency block coding such that the data symbols combined with space frequency mapping and thus affected by the same fading coefficient are not subsequent data symbols in the original data stream. The mapping of the data symbols on the subcarriers for the first antenna corresponds to the classical in verse discrete fourier transformation. The receiver with inverse OFDM operation and space frequency block decoding is shown. The received signals on sub channels $h_{1}$ and $h_{2}$ hasguard interval removal and Fast Fourier transform (FFT) is performed. Through ML detection the symbols are identified and passed through the space frequency decoder. The symbols are then recovered by parallel to serial conversion.

\section{MODULATION}

\section{TECHNIQUEANDANTENNACONFIGURATION}

The choice of modulation scheme plays a very important role. This means that the order of modulation needs to be selected in such a manner that the maximum allowable probability of error is never exceeded. When this is done,

there would be a compromise with the probability of error. Here are three types of modulations used here. These methods are QPSK, 16QAM, 64QAM

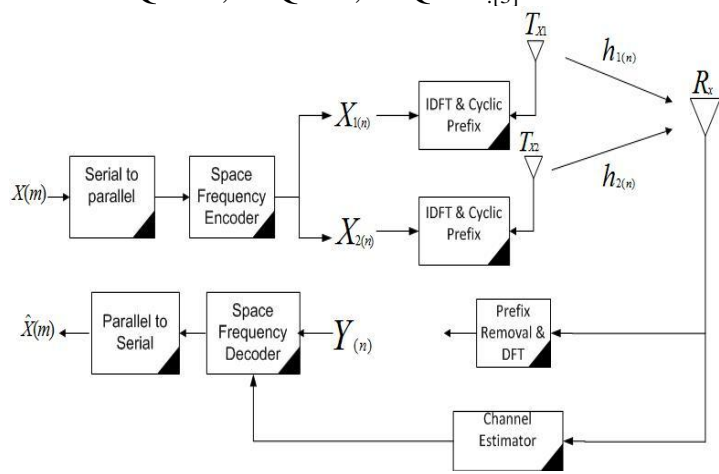

Fig2.2.2: SFBC-OFDM Block Diagram

Here 2x1 STBC-OFDM and 2X2 STBC-OFDM system is discussed in brief. Fig.3.1 shows the 2x1 STBC-OFDM system. It has two transmitter and one receiver antenna. The data is transmitted over 2 links. The arrangement of symbols is according to the Alamouti scheme. Diversity Coding is the spatial coding techniques. Wireless channels severely suffer from fading phenomena, which causes unreliability in data decoding. Diversity coding sends multiple copies through multiple transmit antennas, so as to improve the reliability of the data reception. If one of them fails to receive, the others are used for data decoding. Here in $2 \times 1$ system the diversity order is 2. Fig.3.2 shows the $2 \times 2$ STBC-OFDM systems. The data is transmitted over 4 links having 2 transmitters and 2 receiver antenna. Hence the diversity order is $4\left(2 \mathrm{xN}_{\mathrm{r}}\right)$. Diversity gain is the increase in signal-to-interference ratio due to some diversity scheme, or how much the transmission power can be reduced when a diversity scheme is introduced, without a performance loss. Diversity gain is usually expressed in decibel.

\section{SIMULATION PARAMETERS AND RESULTS}

We consider MIMO-OFDM system with 64 subcarriers and QPSK modulation in presence of AWGN noise. We assume a Rayleigh Fading Channel. The bit-error rate (BER) is evaluated as a function of the SNR per bit.Each pair of transmit-receiver antennas provides a signal path from transmitter to receiver. By sending the same information through different paths, multiple independently-faded replicas of the data symbol can be obtained at the receiver end.

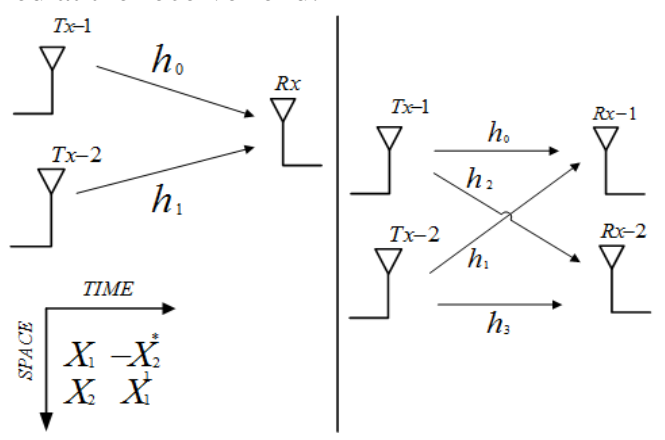

Fig.3.1 2x1 STBC-OFDM Fig.3.2 2x2 STBC-OFDM 
A diversity gain'd' implies that in the high SNR region, my $\mathrm{P}_{\mathrm{e}}$ decays at a rate of $1 / \mathrm{SNR}^{\mathrm{d}}$ as opposed to $1 / \mathrm{SNR}$ for a SISO system ${ }_{[4]}$. The maximal diversity gain $\mathrm{d}_{\max }$ is the total number of independent signal paths that exist between the transmitter and receiver. For an (MR, MT) system, the total number of signal paths is $\mathrm{M}_{\mathrm{R}} \mathrm{M}_{\mathrm{T}}$. Fig.4.1 shows the effect of CFO on MIMO-OFDM system. The comparison is done for 2x1 STBC-OFDM, 2x2 STBCOFDM and 2x1 SFBC-OFDM.

Though 2X2 STBC-OFDM has better performance, it is more sensitive to CFO. Hence, advantage of diversity of $2 \times 2$ antenna configuration is degraded due to presence of CFO. Performance degradation is more for $2 \times 2$ STBCOFDM system Performance is similar for $\mathrm{CFO}$ value above 0.12 for $2 \times 1$ STBC-OFDM and $2 \times 1$ SFBC-OFDM.

\section{CONCLUSION}

The effect of CFO is analysed in MIMO-OFDM system by varying the modulation techniques and different antenna configurations for multi-user. It can be concluded that higher modulation technique is much sensitive to higher values of CFO. STBC-OFDM has better performance compared to SFBC-OFDM for higher modulation technique when no CFO is applied. Diversity is adversely affected by CFO.

\section{ACKNOWLEDGMENT}

This research was supported by Dharmsinh Desai Institute of Technology...I thank my colleague Dipesh Khandhar (M.Tech.) from who provided insight and expertise thatgreatly assisted the research, lthough they may not agree with all of the interpretations/conclusions of this paper. I thank Proff. Hardip K. Shah for assistance with technique, methodology, and for comments that greatly improved the manuscript.

\section{REFERENCES}

[1] Siavash M. Alamouti,A Simple Transmit Diversity Technique For Wireless Communications IEEE Journal On Select Areas In Communications, Vol. 16, No. 8, pp. no. 1451-1458,Oct-1998.

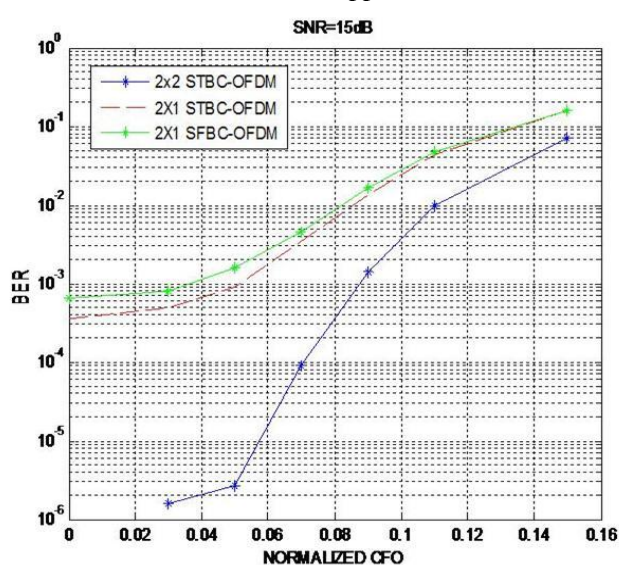

[2] Fig4.1Effect ofCFO on MIMO-OFDM

[2] V. Tarokh, H. Jafarkhani and A. R. CalderbankSpace-time block codes from orthogonal designs IEEE Trans. Inform. Theory, vol. 45, pp. 1456-1467, July 1999.

[3] Banelli, P.Suraweera, H.A.ChauYuen,Performance of Alamouti Space-Time Coded OFDM with Carrier Frequency Offset Global
Telecommunication, Conference Publications, pp. no. 1-5, 9th Dec. 2011.

[4] Ho-Chul Jung, Chang-Ju Kim, Jong-Ho Kim and Hyung-Rae Park, A comparative performance analysis of STBC-OFDM systems under rayleigh fading environments 14th IST Mobile \& Wireless Communication Summit, Dresden, 23rd June 2005.

[5] Manav R. Bhatnagar, R.Vishwanath and VaibhavBhatnagar, Performance Analysis of Space-Time Block Codes in Flat Fading MIMO Channels with Offsets Hindawi Publishing Corporation, EURASIP Journal on Wireless Communications and Networking, Volume, Article ID 30548, 7 pages,10th May-2007 41.

[6] L. Hanzo, Y. Akhtman, M. Jiang, L. Wang, MIMO-OFDM for LTE, WiFi and WiMAX Coherent versus Non-Coherent and Cooperative Turbo-Transceivers 1st published 2011, Wiley-IEEE Press, Print ISBN 9780470686690.

[7] DeepaknathTandurandMarcMoone, STBC MIMO OFDM systems with implementation impairments Vehicular Technology Conference, VTC 2008-Fall IEEE 68th, Conference Publications, pp.no. 1-5, sept.2008.

[8] Jianwu Chen, Yik-ChungWu, Shaodan Ma, and Tung-Sang Ng, Joint CFO and Channel Estimation for Multiuser MIMO-OFDM Systems With Optimal Training Sequences IEEE Transactions On Signal Processing, VOL. 56, NO. 8, pp. no. 4008-4019, Aug 2008.

[9] G.Bauch, Space-time block codes versus space-frequency block codes in Proc. IEEE Vehicular Technology Conference, pp. 567571, May 2003.

[10] K. Lee and D. Williams, A space-frequency transmitter diversity technique for OFDM systems Proc. IEEE GLOBECOM' 2000.

[11] K. F. Lee and D. B. Williams, A space-time coded transmitter diversity technique for frequency selective fading channels in Proc. IEEE SAM 2000, Cambridge, MA, pp. 149-152, Mar. 2000.

\section{BIOGRAPHY}

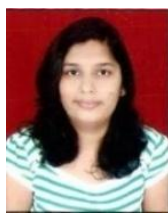

Authors Ankita K. Panchal tireceived the B.E.and M.Tech.degrees in Electronics and Communication engineering from Gujarat University and Dharmsinh Desai university in 2011 and 2013, respectively. She is currently with Sigma Group of institutes as Assistant Professor.Her areas of interest include smart antenna techniques, coding and modulation and wireless communication. 